\title{
Stress and Drug Dependence Differentially Modulate Norepinephrine Signaling in Animals with Varied HPA Axis Function
}

\author{
Megan E Fox', R Isaac Studebaker', Nathaniel J Swofford' and R Mark Wightman*,' \\ 'Department of Chemistry, Center for Neuroscience at the University of North Carolina at Chapel Hill, Chapel Hill, NC, USA
}

\begin{abstract}
Previous work has demonstrated the importance of genetic factors and stress-sensitive circuits in the development of affective disorders. Anxiety and numerous psychological disorders are comorbid with substance abuse, and noradrenergic signaling in the bed nucleus of the stria terminalis (BNST) is thought to be a source of this convergence. Here, we examined the effects of different stressors on behavior and norepinephrine dynamics in the BNST of rat strains known to differ in their HPA-axis function. We compared the effects of acute morphine dependence and social isolation in non-anxious Sprague Dawley (SD) rats, and a depression model, Wistar-Kyoto (WKY) rats. We found a shared phenotype in drug-dependent and singly housed SD rats, characterized by slowed norepinephrine clearance, decreased autoreceptor function, and elevated anxiety. WKY rats exhibited changes in anxiety and autoreceptor function only following morphine dependence. To ascertain the influence of LC inhibition on this plasticity, we administered the LC-terminal-selective toxin DSP-4 to SD and WKY rats. DSP-4-treated SD rats demonstrated a dependence-like phenotype, whereas WKY rats were unchanged. Overall, our findings suggest that individuals with varying stress susceptibilities have different noradrenergic signaling changes in response to stress. These changes may establish conditions that favor stress-induced reinstatement and increase the risk for addiction. Neuropsychopharmacology (20I5) 40, 1752-176I; doi: I0.1038/npp.20I5.23; published online II February 2015
\end{abstract}

\section{INTRODUCTION}

Researchers have demonstrated the importance of central noradrenergic activation in regulating behavioral and physiological responses to stress (Cecchi et al, 2002; Fendt et al, 2005). In part, this is because norepinephrine can engage the hypothalamic-pituitary-adrenal (HPA) axis. Through such actions, norepinephrine has been identified as an important neural substrate associated with the aversive components of morphine withdrawal (Delfs et al, 2000). Such evidence has given support to the view that the negative affect experienced during drug withdrawal is mediated in part by norepinephrine, and can contribute to the addiction cycle (Koob and Volkow, 2010). Interestingly, a number of psychological disorders and addictions are comorbid with stress and involve noradrenergic dysregulation (eg, posttraumatic stress disorder) (Hyman et al, 2006; Sinha, 2008). For example, exposing rats to intruder stress evokes an opiate-dependent like state and alters firing of norepinephrine neurons (Chaijale et al, 2013). Therefore, investigation of noradrenergic systems and their role in the initiation/

* Correspondence: Professor RM Wightman, Department of Chemistry, Center for Neuroscience at the University of North Carolina at Chapel Hill, Campus Box \#3290, Chapel Hill, NC 27599, USA, Tel: +1 919 962 |472, Fax: +919 9622388

E-mail: rmw@unc.edu

Received 18 September 2014; revised 13 January 2015; accepted 14 January 2015; accepted article preview online 20 January 2015 termination of stress is important for understanding the pathophysiology of diseases that co-express with addiction.

The ventral bed nucleus of the stria terminalis (vBNST) is a major target of norepinephrine innervation within the brain (Kilts and Anderson, 1986). Here, forebrain, limbic, and brainstem inputs converge to relay information about both physical and psychological stressors. The BNST receives small noradrenergic input from the locus coeruleus (LC), and major noradrenergic innervation from the $\mathrm{A} 1$ and A2 (via the ventral noradrenergic bundle) cell groups, and projects to stress and reward centers (Forray and Gysling, 2004; Drolet, 2009). The BNST has neurons containing corticotropin-releasing factor (McElligott et al, 2010), excitatory and inhibitory projections to the paraventricular nucleus of the hypothalamus (Choi et al, 2007), and activates the HPA axis (Forray and Gysling, 2004). Norepinephrine is released in the BNST during restraint stress, oral administration of an aversive tastant, and during morphine withdrawal (Fuentealba et al, 2000; Pardon et al, 2002; Park et al, 2012). Thus, norepinephrine release in the BNST can integrate stressful and aversive stimuli to generate an appropriate physiological response.

Previously, we showed that two different rat strains, Sprague-Dawley and Lewis, markedly differed in the response of their noradrenergic system to morphine withdrawal (McElligott et al, 2013). Sprague-Dawley rats demonstrated profound plasticity of uptake and autoreceptor function, whereas control mechanisms were unchanged in Lewis rats. 
To better understand how genetic differences interact with drug withdrawal and stressors, we chose to compare the stress responses of Sprague-Dawley (SD) and Wistar-Kyoto (WKY) rats. WKY rats exhibit increased depressive phenotypes and HPA axis function relative to SpragueDawley rats (Cohen et al, 2006; Carr and Lucki, 2010), and under restraint-stress, extracellular norepinephrine varies between the two (Pardon et al, 2002). Here, we used fastscan cyclic voltammetry to evaluate differences in norepinephrine overflow and regulation between SD and WKY rats in response to stress. We subjected animals to acute morphine-dependence, 2 weeks of social-isolation, and DSP-4 lesioning. In response to these stressors, we found robust neurochemical changes that differed between strains and corresponded with anxiety-like behavior.

\section{METHODS}

\section{Animal Care}

Experiments were performed in accordance with University of North Carolina at Chapel Hill (UNC) Institutional Animal Care and Use Committee's guidelines. Subjects were SpragueDawley and Wistar-Kyoto rats (males, 250-350 g on arrival from Charles River, Wilmington, MA) pair-housed in UNC animal facilities on a 12-hour day/light cycle. Animals were given ad libitum access to food and water, and their health was monitored daily during treatments. For social isolation, after 1-week of acclimation, subjects were randomly split into single or pair-housing for 2 weeks. Care was taken to reduce the number of animals used. For anesthetized voltammetry experiments, 108 Sprague-Dawley and 103 Wistar-Kyoto rats were used. A separate group of 32 Sprague-Dawley and 48 Wistar-Kyoto rats was used for anxiety measures on the Elevated Plus Maze (EPM).

\section{Chemicals and Drugs}

Drugs were purchased from Sigma-Aldrich (St Louis, MO), with the exception of $\alpha_{2 C}$ antagonist JP-1302 dihydrochloride (Tocris Bioscience, Ellisville, MO), dissolved in sterile saline $(0.9 \%)$, and used as received. JP-1302 and $\alpha_{2 \mathrm{~A}}$ antagonist BRL-44408 maleate were administered to naïve animals in a range from 0.2 to $5 \mathrm{mg} / \mathrm{kg}$ i.p. to build a dose-response curve. Treated and control animals were given either $2 \mathrm{mg} / \mathrm{kg}$ BRL44408 i.p. or $2 \mathrm{mg} / \mathrm{kg} \alpha_{2 \mathrm{~A}}$ agonist Guanfacine $\mathrm{HCl}$ i.p. to assay $\alpha_{2 \mathrm{~A}}$ function. At the end of the experiment, animals were given $2 \mathrm{mg} / \mathrm{kg}$ dopamine D2 antagonist raclopride tartrate i.p., followed by $5 \mathrm{mg} / \mathrm{kg} \alpha_{2}$ antagonist idazoxan i.p. to validate signal per Park et al, 2009.

\section{Measurement of Norepinephrine Release}

Norepinephrine release in the vBNST was measured in anesthetized animals as described previously (Park et al, 2009) (Supplementary Methods).

\section{Elevated Plus Maze}

Anxiety-like behavior was measured as described previously (McElligott et al, 2013) (Supplementary Methods). Briefly, the number of entries and time spent in each section of the maze was measured over $5 \mathrm{~min}$. Preference for the open arms was determined in each animal and was expressed as a ratio of open-arm time over closed-arm time.

\section{Morphine Dependence}

For 3 days, rats were administered $10 \mathrm{mg} / \mathrm{kg}$ morphine sulfate s.c. once daily, followed $4 \mathrm{~h}$ later by $1 \mathrm{mg} / \mathrm{kg}$ naloxone $\mathrm{HCl}$ to induce withdrawal. Somatic indices of withdrawal (eg, teeth chattering) were scored each day per Schulteis et al, 1999. Control animals received 3 days of $0.5 \mathrm{ml}$ saline s.c., followed $4 \mathrm{~h}$ later by $1 \mathrm{mg} / \mathrm{kg}$ naloxone. On day 4, drug-free rats were assayed on the EPM or underwent surgery for norepinephrine measurements.

\section{DSP-4 Lesioning}

Wistar-Kyoto and Sprague-Dawley rats $(150-200 \mathrm{~g}$ on arrival) were pair-housed, and administered two i.p., $50 \mathrm{mg} / \mathrm{kg} \quad \mathrm{N}$-(2-chloroethyl)-N-ethyl-2-bromobenzylamine (DSP-4) injections in $1 \mathrm{ml} / \mathrm{kg}$ saline 3 days apart. Control animals were given two $1 \mathrm{ml} / \mathrm{kg}$ i.p. saline injections 3 days apart. Animals were allowed to recover for $>10$ days after the last injection.

\section{Statistics}

Results are presented as average values \pm SEM. Two-way analysis of variance (ANOVA) with post hoc Bonferroni tests were used to determine statistical significance. An unpaired $t$-test was used to determine differences in anxiety between morphine-dependent and control WKY rats. Differences were considered significant when ${ }^{\star} P<0.05$, ${ }^{* *} P<0.01,{ }^{* * *} P<0.005$.

\section{RESULTS}

First, we chose to extend prior work demonstrating straindependent differences in norepinephrine regulation during stress (Pardon et al, 2002; McElligott et al, 2013). We characterized vBNST norepinephrine synaptic function in anesthetized WKY rats with electrical stimulations of the ventral noradrenergic bundle, and measured subsequent norepinephrine overflow with fast-scan cyclic voltammetry (Park et al, 2009; Herr et al, 2012). This technique allows characterization of release and uptake of biogenic amines (John et al, 2006). We measured norepinephrine by placing the carbon-fiber microelectrode directly beneath the anterior commissure (electrode placement shown in Figure 1a, sample color plot encoding voltammetric recordings in Figure 1b). Release increased linearly with increasing stimulation duration in WKY rats $(60 \mathrm{~Hz}$ stimulations, $r^{2}=0.99$, slope: $3.71 \pm 0.24$; Figure $1 \mathrm{c}$ ) similar to our findings in SD rats (slope: $3.11 \pm 0.21$, McElligott et al, 2013). Despite known phenotypic variations (Carr and Lucki, 2010), we found no differences in norepinephrine clearance $\left(t_{1 / 2}\right)$ between WKY and SD rats in a baseline state ( $1.6 \pm 0.12 \mathrm{~s} v s 1.7 \pm 0.10 \mathrm{~s}, n=22$ and 26 , respectively).

The adrenergic $\alpha_{2}$ receptor serves as the inhibitory autoreceptor for norepinephrine, and two differentially regulated subtypes are present in the BNST (Scheinin et al, 1994). We assayed autoreceptor influence on release with $\alpha_{2}$ 
a

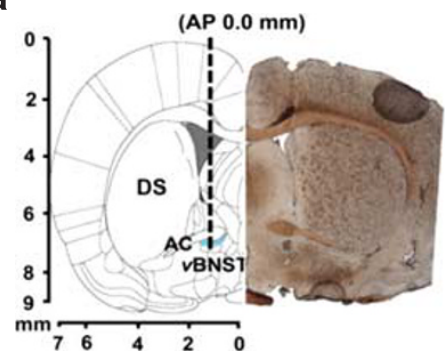

C

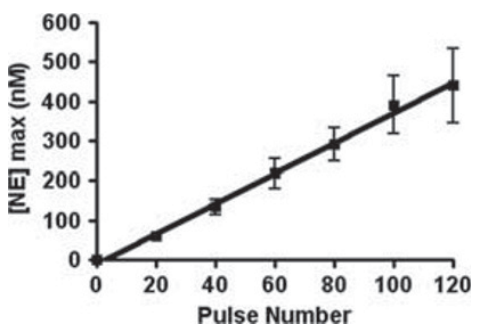

b

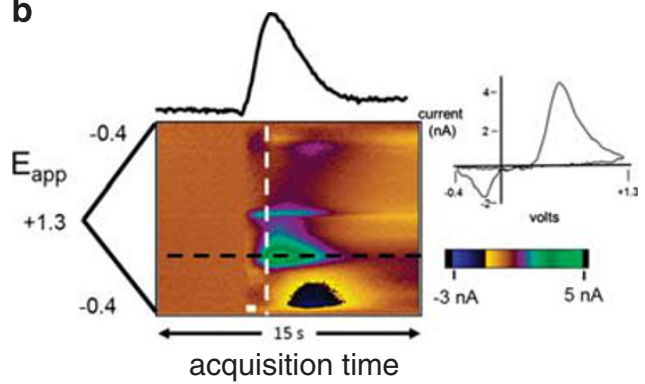

d

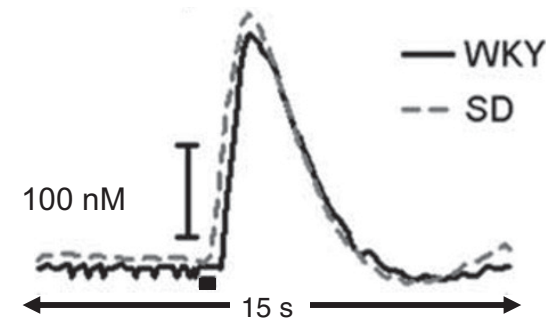

e
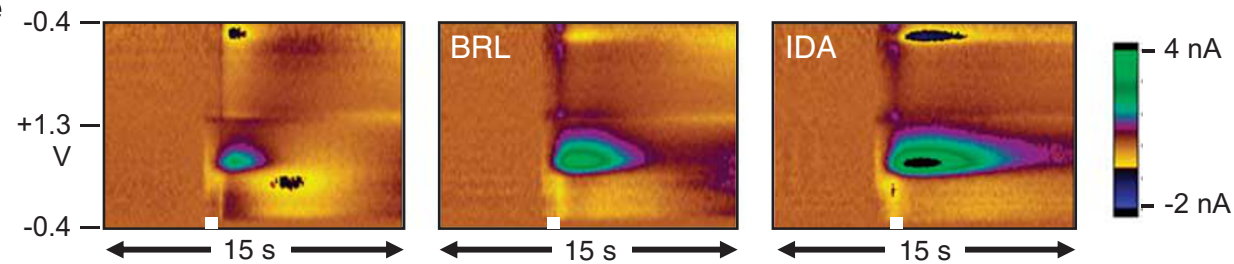

f
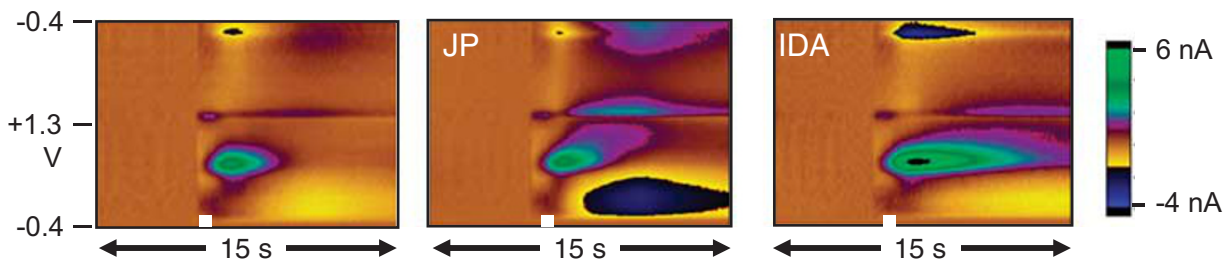

Figure I Fast-Scan Cyclic Voltammetry of norepinephrine in the ventral bed nucleus of the stria terminalis (vBNST). (a) Electrode tract (dashed line) and representative histology of carbon-fiber electrode placement in the vBNST (dashed circle; DS, dorsal striatum; AC, anterior commissure). (b) Norepinephrine measured in the VBNST after electrical stimulation (white bar). The cyclic voltammogram (current vs potential) is obtained from the white dashed line, and the concentration vs time trace from the black dashed line. (c) Input-output curve of [NE] max at 20,40, 60, 80, and I20 stimulation pulses in WKY rats. (d) Representative concentration traces comparing norepinephrine release and uptake in SD and WKY rats. (e and f) Representative color plots of electrically evoked norepinephrine in the vBNST with $\alpha_{2 A}$ (BRL), $\alpha_{2 C}$ (JP), and non-selective $\alpha_{2}$ (IDA) antagonists on board in WKY rats.

antagonist idazoxan in WKY and SD rats. After drug administration $(5 \mathrm{mg} / \mathrm{kg})$, the amplitude in evoked norepinephrine $\left([\mathrm{NE}]_{\max }\right)$ was increased similarly in both strains (WKY: $205 \pm 10.3 \%$ vs SD: $216 \pm 21.1 \%, n=6$ and 5 , respectively). To examine $\alpha_{2}$ subtype-specific effects, we employed $\alpha_{2 \mathrm{~A}}$ and $\alpha_{2 \mathrm{C}}$ selective antagonists BRL-44408 and JP-1302, respectively. We found increased evoked norepinephrine in WKY rats following $2 \mathrm{mg} / \mathrm{kg}$ BRL-44408, but not $2 \mathrm{mg} / \mathrm{kg} \mathrm{JP}-1302$ (examples in Figure 1e and f, BRL: $140 \pm 6.9 \%$ vs JP: $95 \pm 4.5 \%, n=5)$. Similar effects were found in SD rats: (BRL: $157 \pm 15.4 \%$. vs JP: $102 \pm 7.3 \%, n=5$ and 6 , respectively). The selected doses $(2 \mathrm{mg} / \mathrm{kg})$ approach saturation (Supplementary Figure 1) and are sufficient to produce robust behavioral effects in vivo (Sallinen et al, 2007). Thus, our findings indicate the $\alpha_{2 \mathrm{~A}}$ subtype is the principle noradrenergic autoreceptor in the vBNST of both WKY and SD rats, and it exerts similar control over norepinephrine release in both strains.
Norepinephrine Dynamics were Differentially Altered in Morphine-Dependent SD and WKY Rats

We hypothesized that SD and WKY rats would not differ in their response to morphine-dependence, owing to their noradrenergic similarity in a baseline state. To compare noradrenergic plasticity, we established morphine-dependence in SD and WKY rats as before (Schulteis et al, 1999; McElligott et al, 2013). Briefly, rats received $10 \mathrm{mg} / \mathrm{kg}$ morphine followed $4 \mathrm{~h}$ later by $1 \mathrm{mg} / \mathrm{kg}$ naloxone once daily for 3 days. Consistent with our prior work, withdrawal was behaviorally evident in both strains by day 3 (Supplementary Figure 2). On day 4, rats were anesthetized and electrically evoked norepinephrine was recorded. First, we examined the effects on norepinephrine clearance and found clearance half-life showed a main effect of strain $(\mathrm{F}=5.8, P<0.05)$ and treatment $(\mathrm{F}=5.8, P<0.05)$. There was also a significant treatment $\times$ strain interaction (two-way ANOVA, $F=10.35$, 
$P<0.01)$. In agreement with our previous work, morphinedependent SD rats showed impaired uptake relative to controls $(2.2 \pm 0.16 \mathrm{~s}$ vs $1.5 \pm 0.06 \mathrm{~s}, n=12$ and 11 , respectively, $P<0.001)$. To our surprise, uptake was unaltered in WKY rats $(1.5 \pm 0.14 \mathrm{~s} v s 1.6 \pm 0.10 \mathrm{~s}, n=9$ and 10 , respectively, $P>0.05$, Figure 2a). As before, the protocol we employed allows time for clearance of morphine and naloxone (Trescot et al, 2008), thus altered uptake in SD rats is a consequence of morphine withdrawal.

To assay the effects of morphine withdrawal on $\alpha_{2 \mathrm{~A}}$ function in these two strains, we administered $2 \mathrm{mg} / \mathrm{kg}$ of selective antagonist BRL-44408, or agonist guanfacine $(2 \mathrm{mg} / \mathrm{kg}$, GFC) and determined the effects on release. The doses were selected based on dose-response analysis in Supplementary Figure 1 and 3. Response to autoreceptor drugs showed a main effect of morphine treatment (two-way ANOVA, BRL: $\mathrm{F}=26, \quad P<0.0005 ; \mathrm{GFC}: \mathrm{F}=18.2, P<0.005)$ and strain (two-way ANOVA, GFC: $\mathrm{F}=4.7 P<0.05$ ). Post hoc analysis revealed a significant decrease in response to BRL between morphine-dependent animals and their saline-naloxone

a

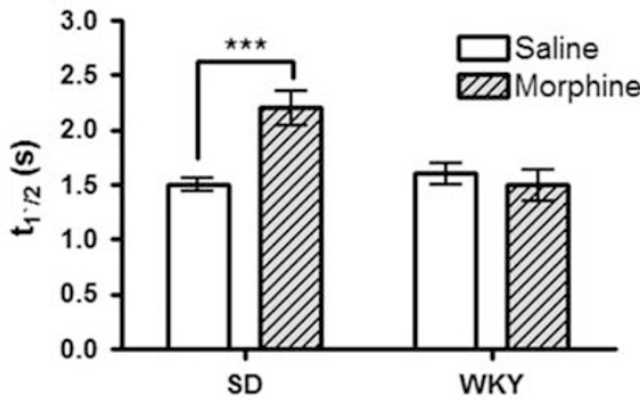

b
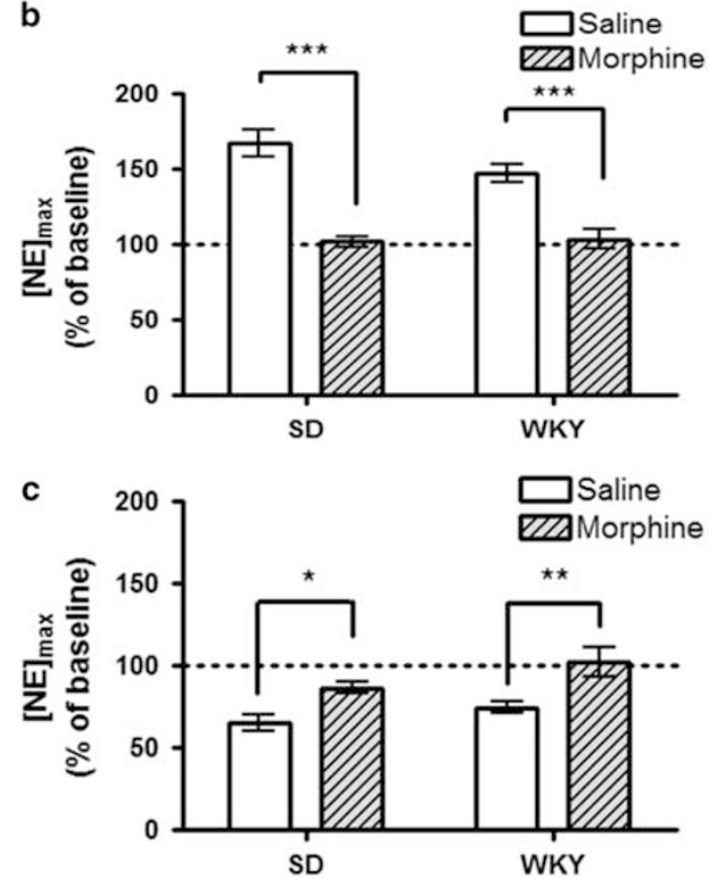

Figure 2 The effects of morphine dependence in Sprague-Dawley and Wistar-Kyoto rats. (a) Average norepinephrine clearance as measured by $t_{1 / 2}$. (b and $c$ ) Average evoked norepinephrine ([NE $]_{\max }$ ) following administration of $2 \mathrm{mg} / \mathrm{kg}$ BRL-44408 (b), or guanfacine (GFC) (c) relative to pre-drug stimulated release. Data are presented as mean $\pm \mathrm{SEM}$. Bonferroni post hoc analysis: $* P<0.05$, $* * P<0.01$, **** $P<0.005$. controls (SD: $102 \pm 3.5 \%$ for morphine withdrawal $v s$ $167 \pm 9.1 \%$ for control, $n=7$, respectively, $P<0.001$; WKY: $104 \pm 6.5 \%$ for morphine withdrawal vs $147 \pm 6.4 \%$ for control, $n=7$, respectively, $P<0.001$, Figure $2 \mathrm{~b}$ ). Additionally, the $\alpha_{2 \mathrm{~A}}$ agonist, GFC, was less effective at inhibiting evoked norepinephrine release in both strains (SD: $87 \pm 3.6 \%$ for morphine withdrawal $v s 65 \pm 4.9 \%$ for control, $n=7$, respectively, $P<0.05$; WKY: $102 \pm 8.9 \%$ for morphine withdrawal vs $75 \pm 3.8 \%$ for control, $n=7$, respectively, $P<0.01$, Figure 2c). Thus, following morphine withdrawal, $\alpha_{2 \mathrm{~A}}$ function is desensitized in both SD and WKY rats.

\section{WKY and SD Rats Exhibited Increased Anxiety-Like Behavior following Morphine-Dependence}

We previously showed that morphine withdrawal increases anxiety-like behavior in SD rats (McElligott et al, 2013). To examine its impact on WKY rats, we assayed their behavior on the EPM. Withdrawal caused WKY rats to become more anxious, as they had reduced preference for the open arms compared with their saline-naloxone controls (unpaired $t$-test, Open/Closed time: $0.05 \pm 0.03$ vs $0.39 \pm 0.12, n=8$, respectively, $P<0.01$, Table 1 ). WKY rats treated with saline-naloxone did not demonstrate a change in open-arm preference relative to naïve animals (Table 2, pair-housed).

\section{Social-Isolation Altered Norepinephrine Signaling in SD, but not WKY Rats}

To further investigate differences between SD and WKY norepinephrine responses, we treated rats with chronic social isolation. This is a passive stressor that removes injection/handling stress, and is suggested to generate depression in rodents (Nestler and Hyman, 2010; Butler et al, 2014). Following 2 weeks of single-housing, rats were anesthetized and evoked norepinephrine was recorded. When comparing norepinephrine uptake between single (S) and pair (P)-housed animals, we found significantly slower uptake in SD-S rats compared with SD-P $(2.3 \pm 0.09 \mathrm{~s} v \mathrm{~s}$ $1.7 \pm 0.10 \mathrm{~s}, n=20$ and 26 , respectively, $P<0.001)$. However, WKY-S did not slow uptake relative to pair-housed controls $(1.6 \pm 0.06 \mathrm{~s}$ vs $1.6 \pm 0.12 \mathrm{~s}, n=21$ and 22 , respectively, $P>0.05$, Figure $3 a)$.

We next compared $\alpha_{2 \mathrm{~A}}$ drug effects between single and pair-housed animals, and found a main effect of housing (two-way ANOVA, BRL: $\mathrm{F}=24.12, P<0.0001$; $\mathrm{GFC}$ : $\mathrm{F}=7.2$, $P<0.05)$ and a housing $\times$ strain interaction $(\mathrm{BRL}: \mathrm{F}=8.2$, $P<0.01$; GFC: $\mathrm{F}=5.4, P<0.05)$. Post hoc analysis revealed that BRL was less effective in increasing norepinephrine in SD-S animals as compared with SD-P (97 $\pm 1.4 \%$ vs $158 \pm$ $11.9 \%, n=7$, respectively, $P<0.001$, Figure $3 \mathrm{~b})$. Similarly, the decrease in release following GFC was attenuated in single-housed animals $(91 \pm 3.2 \%$ vs $64 \pm 7.9 \%, \quad n=7$, respectively, $P<0.01$, Figure $3 c$ ). WKY-S failed to decrease drug response when compared with WKY-P rats (BRL: $124 \pm 8.9 \%$ vs $140 \pm 4.8 \%, n=7$ respectively, $P>0.05$; GFC: $78 \pm 6.4 \%$ vs $76 \pm 2.2 \%, n=7$, respectively, $P>0.05)$.

\section{Social Isolation Induces Anxiety-Like Behavior in SD Rats}

We assayed anxiety-like behavior in singly housed animals on the EPM. Enclosed arm time showed a main effect of 
Table I Anxiety-like Behavior Following Morphine Withdrawal in WKY Rats

\begin{tabular}{lccc}
\hline Morphine dependence & Ratio Open/Closed time & Total entries & Open time \\
\hline Wistar-Kyoto & & & Closed time \\
Saline-Naloxone $(n=8)$ & $0.39 \pm 0.12$ & $6 \pm 1$ & $22 \pm 4 \mathrm{~s}$ \\
Morphine-Naloxone $(n=8)$ & $0.05 \pm 0.03 *$ & $6 \pm 1$ & $6 \pm 3 \mathrm{~s} * 14 \mathrm{~s}$ \\
\hline
\end{tabular}

Total number of entries and time spent in the open and enclosed arms of the elevated plus maze was evaluated. An animal's preference for open arms was expressed as a ratio of open-arm time over closed-arm time. Data are presented as mean \pm SEM. Treated WKY were compared with their controls using an unpaired $t$-test. Starred values denote significance between the treated group and the control directly above it. $* P<0.05$.

Table 2 Anxiety-like Behavior Following Differing Stressors

\begin{tabular}{|c|c|c|c|c|}
\hline & Ratio Open/Closed time & Total entries & Open time & Closed time \\
\hline \multicolumn{5}{|l|}{ Social isolation } \\
\hline \multicolumn{5}{|l|}{ Sprague-Dawley } \\
\hline Pair-housed $(n=7)$ & $0.33 \pm 0.09$ & $14 \pm 1$ & $49 \pm 11 s$ & $163 \pm 10 s$ \\
\hline Single-housed $(n=8)$ & $0.12 \pm 0.05 *$ & $12 \pm 2$ & $20 \pm 7 s^{*}$ & $201 \pm 12 s^{*}$ \\
\hline Pair-housed $(n=8)$ & $0.26 \pm 0.06$ & $9 \pm 2$ & $38 \pm 8 s$ & $156 \pm 10 \mathrm{~s}$ \\
\hline Single-housed $(n=8)$ & $0.23 \pm 0.04$ & $9 \pm 1$ & $32 \pm 6 s$ & $148 \pm 9 s$ \\
\hline \multicolumn{5}{|l|}{ Coerulean Lesioning } \\
\hline \multicolumn{5}{|l|}{ Sprague-Dawley } \\
\hline DSP-4 lesioned $(n=7)$ & $0.13 \pm 0.03$ & $7 \pm 3$ & $24 \pm 4 s$ & $193 \pm 14 s$ \\
\hline
\end{tabular}

Total number of entries and time spent in the open and enclosed arms of the elevated plus maze was evaluated. An animal's preference for open arms was expressed as a ratio of open-arm time over closed-arm time. Data are presented as mean \pm SEM. Treated animals were compared with their controls using a two-way ANOVA with Bonferroni post hoc analysis. Differences in anxiety following stress were determined for each strain by comparing treated animals with their respective controls. Starred values denote significance between the treated group and the control directly above it. $* P<0.05$.

strain (two-way ANOVA, $\mathrm{F}=8.24, \quad P<0.01$ ) and a housing $\times$ strain interaction $(\mathrm{F}=4.89, P<0.05)$. Open arm time showed a main effect of housing $(\mathrm{F}=4.83, P<0.05)$. Total number of entries showed a main effect of strain $(\mathrm{F}=6.11, P<0.05)$, and the reduced number of WKY entries agrees with previous studies (Cohen et al, 2006; Carr and Lucki, 2010). Post hoc analysis revealed a significant reduction in open arm preference in single-housed animals (Open time/Closed time: $0.33 \pm 0.09$ vs $0.12 \pm 0.05$, SD-S vs SD-P, $n=8$ and 7 , respectively, $P<0.05)$. WKY-S did not increase anxiety-like behavior relative to WKY-P rats (Table 2).

\section{Coerulean Lesion Induced Noradrenergic Plasticity in SD Rats, but not WKY}

As both morphine dependence and social stress can alter the firing rate of the LC (Chaijale et al, 2013; Van Bockstaele and Valentino, 2013), we wanted to mimic the effects of long-term LC inhibition on BNST norepinephrine and behavior. We lesioned coerulean norepinephrine terminals using the neurotoxin DSP-4, which reduces norepinephrine from LC but not medullary cells (Fritschy and Grzanna, 1989). The vBNST receives little LC input, and stimulation electrode placement in the ventral noradrenergic bundle targets axons primarily from A1/A2 cell groups. Not surprisingly, $[\mathrm{NE}]_{\max }$ was unchanged by DSP-4 treatment (SD: $219 \pm 37 \mathrm{nM}$ vs $322 \pm 87 \mathrm{nM}, n=7$, and 6 , respectively, $P>0.05$; WKY: $230 \pm 23 \mathrm{nM}$ vs $239 \pm 19 \mathrm{nM}, n=7$ and 5, respectively, $P>0.05)$. However, differences in synaptic function were found between vehicle and DSP-4-treated SD rats. Clearance half-life showed a strain $\times$ treatment interaction $(\mathrm{F}=9.5, P<0.01)$ and main effect of strain $(\mathrm{F}=13.2$, $P<0.005)$. DSP-4-treated SD rats had slower uptake than their controls $(2.6 \pm 0.27 \mathrm{~s} v s 1.6 \pm 0.15 \mathrm{~s}, n=7$ and 8 , respectively, $P<0.01)$. WKY rats were unchanged $(1.1 \pm 0.23 \mathrm{~s} v \mathrm{~s}$ $1.5 \pm 0.22 \mathrm{~s}, n=5$ and 8 , respectively, $P>0.05$, Figure $4 \mathrm{a})$. Response to both BRL and GFC showed a strain $\times$ treatment interaction (two-way ANOVA, BRL: $\mathrm{F}=6.7, P<0.05$; GFC: $\mathrm{F}=4.5, \quad P<0.05)$, and main effect of treatment (BRL: $\mathrm{F}=11.9, P<0.01$; GFC: $\mathrm{F}=5.8, P<0.05)$. Post hoc analysis revealed the response to BRL and GFC was blunted in DSP-4 
a

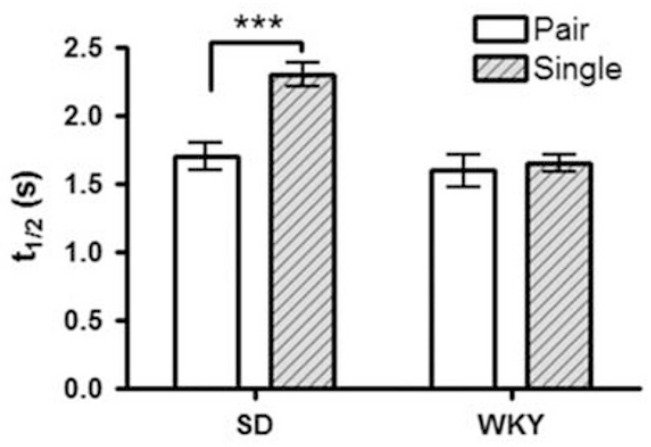

b
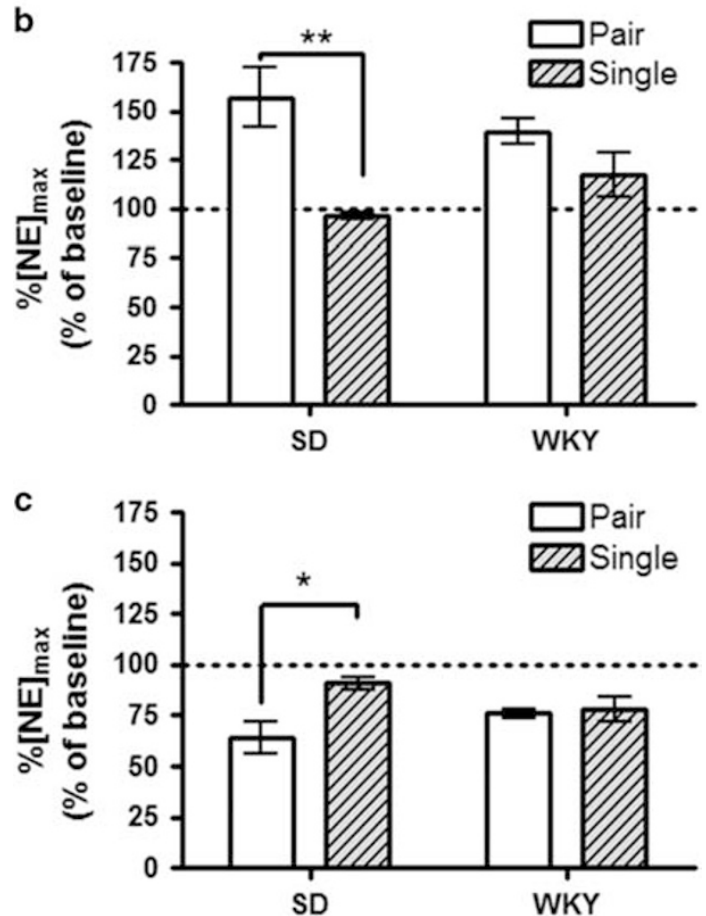

Figure 3 The effects of social isolation in Sprague-Dawley and WistarKyoto rats. (a) Average norepinephrine clearance as measured by $t_{1 / 2}$. (b and c) Average evoked norepinephrine ( $[\mathrm{NE}]_{\max }$ ) following administration of $2 \mathrm{mg} / \mathrm{kg}$ BRL-44408 (b), or guanfacine (GFC) (c) relative to pre-drug stimulated release. Data are presented as mean \pm SEM. Bonferroni post hoc analysis: $* P<0.05$, $* * P<0.01$, $* * * P<0.005$.

treated SD rats (BRL: $100 \pm 5.3 \%$ vs $155 \pm 14.7 \%, n=5$, respectively, $P<0.05$; GFC: $90 \pm 2.8 \%$ vs $64 \pm 7.9 \%, n=5$ and 7 , respectively, $P<0.01$ ) but not WKY rats (BRL: $130 \pm 6.4 \%$ vs $138 \pm 7.2 \% \quad n=5$, respectively, $P>0.05$; GFC: $80 \pm 3.8 \%$ vs $78 \pm 1.3 \% n=5$, respectively, $P>0.05$, Figure 4c).

\section{Coerulean Lesion Increased Anxiety-Like Behavior in SD Rats}

The impact of DSP-4 lesioning on anxiety is dependent on dosage, recovery, and housing (Harro et al, 1995; Lapiz et al, 2001; Itoi et al, 2011). Our treatment increased anxiety-like behavior in SD rats, but not WKY rats as measured on the EPM. DSP-4-treated SD rats spent less time in the open arms, and more time in the enclosed arms (Open time/Closed time: $0.12 \pm 0.03$ vs $0.26 \pm 0.01, n=8$, a
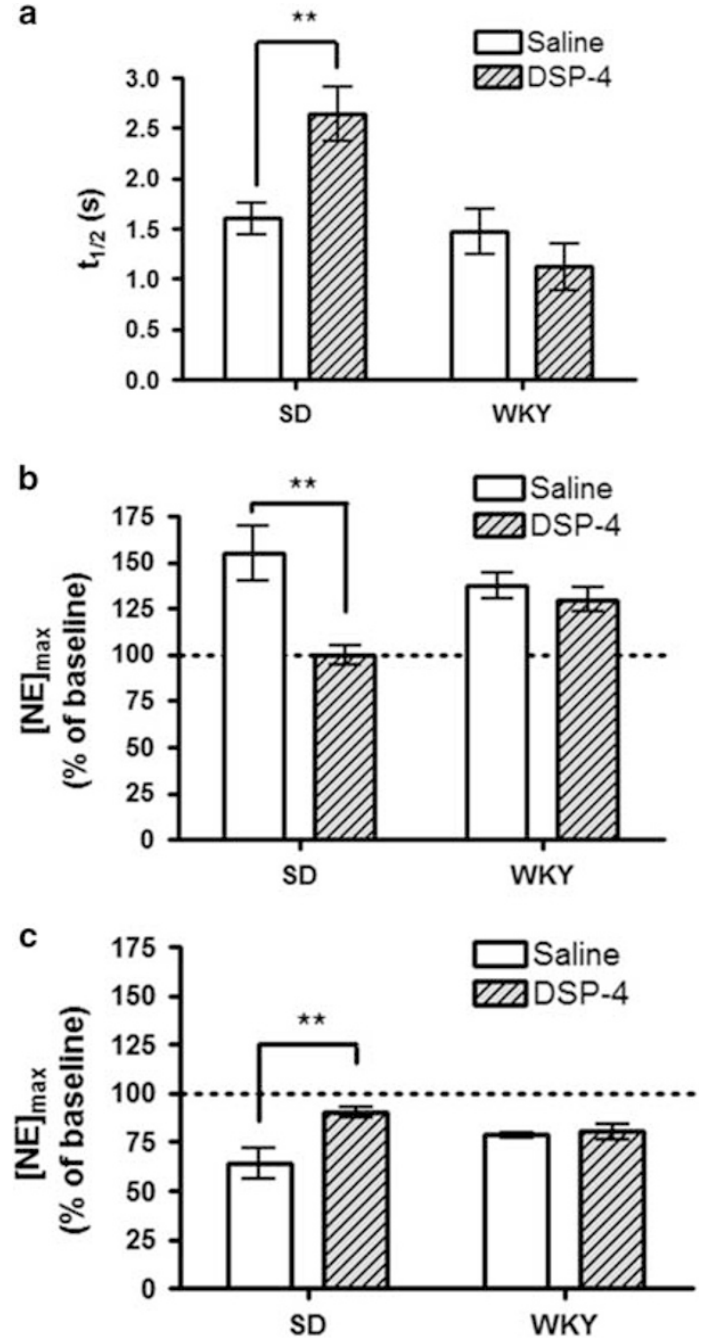

Figure 4 The effects of coerulean lesioning in Sprague-Dawley and Wistar-Kyoto rats. (a) Average norepinephrine clearance as measured by $t_{1 / 2}$. (b and c) Average evoked norepinephrine ([NE] $\max$ ) following administration of $2 \mathrm{mg} / \mathrm{kg}$ BRL-44408 (b) or guanfacine (GFC) (c) relative to pre-drug stimulated release. Data are presented as mean \pm SEM. Bonferroni post hoc analysis: ${ }^{*} * P<0.01$.

DSP-4 $v s$ saline, respectively, $P<0.05$. The number of entries was also reduced ( $6 \pm 2$ vs $15 \pm 2, n=8$, respectively, $P<0.05)$. DSP-4-treated WKY rats did not show increased anxiety compared with controls (Table 2).

\section{DISCUSSION}

Extracellular neurotransmitter concentrations are balanced by release and uptake processes (Wightman et al, 1988). Norepinephrine release is controlled by both cell firing and autoreceptor modulation, and the norepinephrine transporter is the primary clearance mechanism (Xu et al, 2000) with metabolism operating on a slower time scale (Near et al, 1988). Previously, we showed Lewis rats have hindered uptake, blunted autoreceptor function (McElligott et al, 2013), and increased anxiety compared with SD rats. When 
stressed with morphine withdrawal, Lewis rats showed no change in the regulation of BNST norepinephrine. This was in contrast to the dramatic alterations in norepinephrine clearance rate and autoreceptor function found in drugdependent SD rats (McElligott et al, 2013). Here, we show that naïve SD and WKY rats are indistinguishable from each other with respect to evoked norepinephrine overflow, however, each strain diverges in their adaptations to stress. We found that following morphine withdrawal, WKY rats were similar to SD rats with increased anxiety and attenuated autoreceptor function, however, norepinephrine uptake rate in WKY rats was unaltered. Additionally, BNST norepinephrine control mechanisms were unchanged in socially isolated WKY rats, whereas SD rats challenged with social isolation became more anxious and exhibited exacerbated norepinephrine signaling. Following lesions of norepinephrine in the LC, SD rats showed reduced norepinephrine control and increased anxiety, surprisingly similar to acute morphine dependence, but WKY rats were unaffected. Overall, we find WKY rats respond only to select stressors and that anxiety correlates with the degree of regulation of extracellular norepinephrine in the BNST.

The $\alpha_{2}$ receptors are the primary adrenergic autoreceptors, and they show subtype-specific desensitization and phosphorylation by G-protein receptor kinases (Jewell-Motz and Liggett, 1996). As two subtypes of $\alpha_{2}$ receptors are expressed in the BNST (Scheinin et al, 1994), we paired fast-scan cyclic voltammetry measurements with receptorsubtype-specific pharmacology to assay control over norepinephrine release by each subtype. Knock-out mice were used to determine that $\alpha_{2 \mathrm{~A}}$ acts as the principle autoreceptor (Trendelenburg et al, 2001). In agreement with this, we found the inhibition of $\alpha_{2 \mathrm{~A}}$ increased norepinephrine overflow in the vBNST to a similar extent in both rat strains. Although blockade of $\alpha_{2 \mathrm{C}}$ receptors did not increase norepinephrine overflow, $\alpha_{2 C}$ inhibition generated a large alkaline flux following stimulated norepinephrine release, seen in Figure if as blue current just below norepinephrine peak oxidation potential. The $\alpha_{2 \mathrm{C}}$ receptors in the vBNST may therefore be positioned to regulate blood flow/ metabolism in the vBNST (Bucher et al, 2014).

WKY rats exhibit reduced locomotion in an open field, limited exploration in the EPM, low baseline startle, and limited stress responsivity as compared with SD rats (Pardon et al, 2002; Cohen et al, 2006). Correspondingly, increases in extracellular norepinephrine in the BNST, evaluated by microdialysis, were greater in SD during restraint stress than WKY rats (Pardon et al, 2002). Following the stresses of both social isolation and morphine withdrawal, we found in SD rats that uptake and autoreceptor regulation are downregulated. The decreased noradrenergic control in SD rats could generate the comparatively larger increase in extracellular norepinephrine found with microdialysis. In WKY rats, morphine withdrawal produced only attenuated autoreceptor function, whereas social stress had no effect on norepinephrine control mechanisms. These limited adaptations would result in the smaller alteration in the level of extracellular norepinephrine induced by stress (Pardon et al, 2002). Importantly, cellular activation in the BNST during stress is similar between the two strains ( $\mathrm{Ma}$ and Morilak, 2004). Thus, the greater norepinephrine overflow others have measured in stressed SD likely reflect the changes in uptake rate and desensitized autoreceptors revealed in this work.

Non-specific organic cation transporters (OCTs) are expressed throughout the BNST (Gasser et al, 2009). The high-capacity, low-affinity OCT3 is thought to act as a secondary means of norepinephrine clearance, and is inhibited by physiological levels of corticosterone (Gasser et al, 2006). The extent to which BNST norepinephrine is taken up by OCTs in vivo is not currently known, however, NET knockout mice still demonstrate catecholamine clearance in brain slices (Xu et al, 2000) relative to dopamine transporter knockouts (Giros et al, 1996), indicating this may be a significant clearance mechanism for norepinephrine. Previously, we showed the altered norepinephrine clearance in SD rats following withdrawal was not due to decreased NET binding sites (McElligott et al, 2013). Instead, stress-induced corticosterone release may inhibit OCT3 and reduce clearance rate. WKY rats have elevated peak diurnal levels of corticosterone relative to SD rats (Rittenhouse et al, 2002), which may eliminate any OCT3 component of norepinephrine uptake because of chronic inhibition. In addition, WKY rats exhibit behavioral sensitivity to the NET inhibitor desipramine (Lopez-Rubalcava and Lucki, 2000), highlighting the importance of NET in WKY rats. The unaltered norepinephrine uptake in WKY rats corresponds with their low stress responsivity, and is likely the result of HPA axis dysfunction.

Social stressors promote drug self-administration and escalation, generate a long-lasting tolerance to opiate analgesia, and are as efficacious as physical stress at reinstating morphine place preference (Miczek et al, 2004; Ribeiro Do Couto et al, 2006; Butler et al, 2014). Exposure to stressful life events and HPA axis dysfunction have been implicated in the development of several psychiatric disorders that are comorbid with addiction, however, stress alone is not sufficient for their development (Faravelli et al, 2012). It has been suggested that stress can interact with genetic vulnerabilities in predisposed individuals to create the psychopathology. Valentino and coworkers(Chaijale et al, 2013) found stress activation of the endogenous opioid system sufficient to generate a cellular opiate dependence in SD rats. Here, we used chronic social isolation, a passive stressor suggested to induce depression and anxiety in rodents (Nestler and Hyman, 2010; Butler et al, 2014). Remarkably, after SD rats were socially isolated for 2 weeks, they resembled morphine-dependent SD rats, with increased anxiety and enhanced noradrenergic signaling in the vBNST. Moreover, following social isolation, SD rats anxiety and norepinephrine regulation resembled that of our previous work in Lewis rats (McElligott et al, 2013), a model of increased drug-intake and PTSD (Cohen et al, 2006; Sanchez-Cardoso et al, 2007; Picetti et al, 2012). In stark contrast, we found that WKY rats were unresponsive to social isolation, exhibiting no changes in anxiety-like behavior or norepinephrine signaling relative to their controls. These results support the idea that genetic differences can predispose individuals to psychological disorders or addictions, as their noradrenergic system may already resemble a drug-dependent or anxious state. Additionally, individuals with anxiety or depression may not be able to appropriately adapt to stress because of low responsivity of the noradrenergic system. 
During stress, LC activity is tuned by a balance of CRF excitation, and endogenous opioid inhibition (Van Bockstaele and Valentino, 2013), and chronic stress can decrease LC discharge rates (Chaijale et al, 2013). The BNST receives a small input from the LC (Forray and Gysling, 2004), and its activation may be influenced by altered coerulean discharge rates following stress. Thus, we chose to mimic stressinduced LC inhibition by lesioning it with the potent and selective neurotoxin DSP-4. DSP-4 induces degradation of norepinephrine axons arising from the LC, while leaving brainstem norepinephrine innervation intact (Fritschy and Grzanna, 1989). The behavioral effects of DSP-4 treatment are variable (Harro et al, 1995; Lapiz et al, 2001; Itoi et al, 2011), and in our study, we found an anxiogenic effect in SD rats with no change in WKY rats relative to their respective controls. This LC inhibition also lowered the number of maze entries SD rats made to that of WKY. Surprisingly, the DSP-4 treatment produced a robust, dependence-like phenotype in the BNST norepinephrine regulation of SD rats. Such a response was not expected owing to the limited LC innervation of the BNST. These results may reflect feedback between the two main sources of central noradrenergic innervation through a common afferent (Van Bockstaele and Valentino, 2013) and should be a point of future study. Cross-talk between noradrenergic inputs is supported by work demonstrating the importance of both medullary and coerulean norepinephrine in mediating the aversiveness (Delfs et al, 2000) and somatic withdrawal signs of withdrawal (Maldonado, 1997). Importantly, LC activation during opiate withdrawal is partly a result of excitatory input from the nucleus paragigantocellularis, a structure that innervates the nucleus of the solitary tract (A2). Following DSP-4 treatment, we found no change in norepinephrine regulation in WKY rats, similar to WKY rats exposed to social isolation stress. However, WKY rats were responsive to morphine withdrawal, the aversiveness of which is due to medullary norepinephrine (Delfs et al, 2000). WKY rats overexpress kappa opioid receptors in the LC, leaving the LC in a chronically inhibited state (Carr and Lucki, 2010), a possible explanation for the low number of maze entries and lack of DSP-4 response. This persistent inhibition may attenuate any excitatory influence of CRF and partially explain the lack of noradrenergic facilitation to social isolation. Overall, these results suggest a plasticity of medullary inputs but not LC inputs in the BNST of WKY rats.

We have shown that certain stressors permit exacerbated BNST signaling that is accompanied by increased anxiety. Furthermore, we showed the signaling changes coincide with $\alpha_{2 \mathrm{~A}}$ receptor function and are dependent on rat strain. Social isolation and persistent coerulean inhibition caused dramatic changes in the SD rat, and generated a morphinedependence-like phenotype. WKY rats were unresponsive to both social isolation and DSP-4 treatment. Following morphine withdrawal, they demonstrated increased anxiety and an intermediate change in norepinephrine signaling: decreased $\alpha_{2 \mathrm{~A}}$ function without a change in norepinephrine clearance. Taken together, this differential responsivity may reflect separate noradrenergic mechanisms for adaptation that depend on the stressor or its intensity. Moreover, our data support the idea that genetic factors contribute to stress response, which may in turn generate cellular conditions that favor drug use and future substance abuse issues.

\section{FUNDING AND DISCLOSURE}

The authors declare no conflict of interest.

\section{ACKNOWLEDGEMENTS}

We thank Dr Ryan Vetrano, Dr Fulton Crews, and Dr Zoe McElligott for behavioral assistance and Dr Elizabeth Bucher for comments on an earlier version of this manuscript. We also acknowledge support from the UNC Electronics Facility. This research was supported by an NIH grant to RMW. (NS 015841).

\section{REFERENCES}

Bucher ES, Fox ME, Kim L, Kirkpatrick DC, Rodeberg NT, Belle AM et al (2014). Medullary norepinephrine neurons modulate local oxygen concentrations in the bed nucleus of the stria terminalis. J Cereb Blood Flow Metab 34: 1128-1137.

Butler TR, Ariwodola OJ, Weiner JL (2014). The impact of social isolation on HPA axis function, anxiety-like behaviors, and ethanol drinking. Front Integr Neurosci 7: 102.

Carr GV, Lucki I (2010). Comparison of the kappa-opioid receptor antagonist DIPPA in tests of anxiety-like behavior between Wistar Kyoto and Sprague Dawley rats. Psychopharmacology (Berl) 210: 295-302.

Cecchi M, Khoshbouei H, Javors M, Morilak DA (2002). Modulatory effects of norepinephrine in the lateral bed nucleus of the stria terminalis on behavioral and neuroendocrine responses to acute stress. Neuroscience 112: 13-21.

Chaijale NN, Curtis AL, Wood SK, Zhang XY, Bhatnagar S, Reyes BA et al (2013). Social stress engages opioid regulation of locus coeruleus norepinephrine neurons and induces a state of cellular and physical opiate dependence. Neuropsychopharmacology 38: 1833-1843.

Choi DC, Furay AR, Evanson NK, Ostrander MM, Ulrich-Lai YM, Herman JP (2007). Bed nucleus of the stria terminalis subregions differentially regulate hypothalamic-pituitary-adrenal axis activity: implications for the integration of limbic inputs. J Neurosci 27: 2025-2034.

Cohen H, Zohar J, Gidron Y, Matar MA, Belkind D, Loewenthal U et al (2006). Blunted HPA axis response to stress influences susceptibility to posttraumatic stress response in rats. Biol Psychiatry 59: 1208-1218.

Delfs JM, Zhu Y, Druhan JP, Aston-Jones G (2000). Noradrenaline in the ventral forebrain is critical for opiate withdrawal-induced aversion. Nature 403: 430-434.

Drolet G (2009). Progress in Neuro-Psychopharmacology and Biological Psychiatry. Elsevier: Amsterdam 33: 1289-1586.

Faravelli C, Lo Sauro C, Lelli L, Pietrini F, Lazzeretti L, Godini L et al (2012). The role of life events and HPA axis in anxiety disorders: a review. Curr Pharm Des 18: 5663-5674.

Fendt M, Siegl S, Steiniger-Brach B (2005). Noradrenaline transmission within the ventral bed nucleus of the stria terminalis is critical for fear behavior induced by trimethylthiazoline, a component of fox odor. J Neurosci 25: 5998-6004.

Forray MI, Gysling K (2004). Role of noradrenergic projections to the bed nucleus of the stria terminalis in the regulation of the hypothalamic-pituitary-adrenal axis. Brain Res Brain Res Rev 47: 145-160. 
Fritschy JM, Grzanna R (1989). Immunohistochemical analysis of the neurotoxic effects of DSP-4 identifies two populations of noradrenergic axon terminals. Neuroscience 30: 181-197.

Fuentealba JA, Forray MI, Gysling K (2000). Chronic morphine treatment and withdrawal increase extracellular levels of norepinephrine in the rat bed nucleus of the stria terminalis. J Neurochem 75: 741-748.

Gasser PJ, Lowry CA, Orchinik M (2006). Corticosterone-sensitive monoamine transport in the rat dorsomedial hypothalamus: potential role for organic cation transporter 3 in stress-induced modulation of monoaminergic neurotransmission. J Neurosci 26: 8758-8766.

Gasser PJ, Orchinik M, Raju I, Lowry CA (2009). Distribution of organic cation transporter 3 , a corticosterone-sensitive monoamine transporter, in the rat brain. J Comp Neurol 512: 529-555.

Giros B, Jaber M, Jones SR, Wightman RM, Caron MG (1996). Hyperlocomotion and indifference to cocaine and amphetamine in mice lacking the dopamine transporter. Nature 379: 606-612.

Harro J, Oreland L, Vasar E, Bradwejn J (1995). Impaired exploratory behaviour after DSP-4 treatment in rats: implications for the increased anxiety after noradrenergic denervation. Eur Neuropsychopharmacol 5: 447-455.

Herr NR, Park J, McElligott ZA, Belle AM, Carelli RM, Wightman RM (2012). In vivo voltammetry monitoring of electrically evoked extracellular norepinephrine in subregions of the bed nucleus of the stria terminalis. J Neurophysiol 107: 1731-1737.

Hyman SE, Malenka RC, Nestler EJ (2006). Neural mechanisms of addiction: the role of reward-related learning and memory. Annu Rev Neurosci 29: 565-598.

Itoi K, Sugimoto N, Suzuki S, Sawada K, Das G, Uchida K et al (2011). Targeting of locus ceruleus noradrenergic neurons expressing human interleukin-2 receptor alpha-subunit in transgenic mice by a recombinant immunotoxin anti-Tac(Fv)PE38: a study for exploring noradrenergic influence upon anxiety-like and depression-like behaviors. J Neurosci 31: 6132-6139.

Jewell-Motz EA, Liggett SB (1996). G protein-coupled receptor kinase specificity for phosphorylation and desensitization of alpha2-adrenergic receptor subtypes. J Biol Chem 271: 18082-18087.

John CE, Budygin EA, Mateo Y, Jones SR (2006). Neurochemical characterization of the release and uptake of dopamine in ventral tegmental area and serotonin in substantia nigra of the mouse. J Neurochem 96: 267-282.

Kilts CD, Anderson CM (1986). The simultaneous quantification of dopamine, norepinephrine and epinephrine in micropunched rat brain nuclei by on-line trace enrichment HPLC with electrochemical detection: Distribution of catecholamines in the limbic system. Neurochem Int 9: 437-445.

Koob GF, Volkow ND (2010). Neurocircuitry of addiction. Neuropsychopharmacology 35: 217-238.

Lapiz MD, Mateo Y, Durkin S, Parker T, Marsden CA (2001). Effects of central noradrenaline depletion by the selective neurotoxin DSP-4 on the behaviour of the isolated rat in the elevated plus maze and water maze. Psychopharmacology (Berl) 155: 251-259.

Lopez-Rubalcava C, Lucki I (2000). Strain differences in the behavioral effects of antidepressant drugs in the rat forced swimming test. Neuropsychopharmacology 22: 191-199.

Ma S, Morilak DA (2004). Induction of FOS expression by acute immobilization stress is reduced in locus coeruleus and medial amygdala of Wistar-Kyoto rats compared to Sprague-Dawley rats. Neuroscience 124: 963-972.

Maldonado R (1997). Participation of noradrenergic pathways in the expression of opiate withdrawal: biochemical and pharmacological evidence. Neurosci Biobehav Rev 21: 91-104.

McElligott ZA, Fox ME, Walsh PL, Urban DJ, Ferrel MS, Roth BL et al (2013). Noradrenergic synaptic function in the bed nucleus of the stria terminalis varies in animal models of anxiety and addiction. Neuropsychopharmacology 38: 1665-1673.

McElligott ZA, Klug JR, Nobis WP, Patel S, Grueter BA, Kash TL et al (2010). Distinct forms of Gq-receptor-dependent plasticity of excitatory transmission in the BNST are differentially affected by stress. Proc Natl Acad Sci U S A 107: 2271-2276.

Miczek KA, Covington HE 3rd, Nikulina EM Jr., Hammer RP (2004). Aggression and defeat: persistent effects on cocaine selfadministration and gene expression in peptidergic and aminergic mesocorticolimbic circuits. Neurosci Biobehav Rev 27: 787-802.

Near JA, Bigelow JC, Wightman RM (1988). Comparison of uptake of dopamine in rat striatal chopped tissue and synaptosomes. J Pharmacol Exp Ther 245: 921-927.

Nestler EJ, Hyman SE (2010). Animal models of neuropsychiatric disorders. Nat Neurosci 13: 1161-1169.

Pardon MC, Gould GG, Garcia A, Phillips L, Cook MC, Miller SA et al (2002). Stress reactivity of the brain noradrenergic system in three rat strains differing in their neuroendocrine and behavioral responses to stress: implications for susceptibility to stress-related neuropsychiatric disorders. Neuroscience 115: 229-242.

Park J, Kile BM, Wightman RM (2009). In vivo voltammetric monitoring of norepinephrine release in the rat ventral bed nucleus of the stria terminalis and anteroventral thalamic nucleus. Eur J Neurosci 30: 2121-2133.

Park J, Wheeler RA, Fontillas K, Keithley RB, Carelli RM, Wightman RM (2012). Catecholamines in the bed nucleus of the stria terminalis reciprocally respond to reward and aversion. Biol Psychiatry 71: 327-334.

Picetti R, Caccavo JA, Ho A, Kreek MJ (2012). Dose escalation and dose preference in extended-access heroin self-administration in Lewis and Fischer rats. Psychopharmacology (Berl) 220: 163-172.

Ribeiro Do Couto B, Aguilar MA, Manzanedo C, Rodriguez-Arias M, Armario A, Minarro J (2006). Social stress is as effective as physical stress in reinstating morphine-induced place preference in mice. Psychopharmacology (Berl) 185: 459-470.

Rittenhouse PA, Lopez-Rubalcava C, Stanwood GD, Lucki I (2002). Amplified behavioral and endocrine responses to forced swim stress in the Wistar-Kyoto rat. Psychoneuroendocrinology 27: 303-318.

Sallinen J, Hoglund I, Engstrom M, Lehtimaki J, Virtanen R, Sirvio J et al (2007). Pharmacological characterization and CNS effects of a novel highly selective alpha2C-adrenoceptor antagonist JP-1302. Br J Pharmacol 150: 391-402.

Sanchez-Cardoso P, Higuera-Matas A, Martin S, del Olmo N, Miguens M, Garcia-Lecumberri C et al (2007). Modulation of the endogenous opioid system after morphine self-administration and during its extinction: a study in Lewis and Fischer 344 rats. Neuropharmacology 52: 931-948.

Scheinin M, Lomasney JW, Hayden-Hixson DM, Schambra UB, Caron MG, Lefkowitz RJ et al (1994). Distribution of alpha 2adrenergic receptor subtype gene expression in rat brain. Brain Res Mol Brain Res 21: 133-149.

Schulteis G, Heyser CJ, Koob GF (1999). Differential expression of response-disruptive and somatic indices of opiate withdrawal during the initiation and development of opiate dependence. Behav Pharmacol 10: 235-242.

Sinha R (2008). Chronic stress, drug use, and vulnerability to addiction. Ann NY Acad Sci 1141: 105-130.

Trendelenburg AU, Klebroff W, Hein L, Starke K (2001). A study of presynaptic alpha2-autoreceptors in alpha2A/D-, alpha2B- and alpha2C-adrenoceptor-deficient mice. Naunyn Schmiedebergs Arch Pharmacol 364: 117-130.

Trescot AM, Datta S, Lee M, Hansen H (2008). Opioid pharmacology. Pain Physician 11: S133-S153. 
Van Bockstaele EJ, Valentino RJ (2013). Neuropeptide regulation of the locus coeruleus and opiate-induced plasticity of stress responses. Adv Pharmacol 68: 405-420.

Wightman RM, Amatore C, Engstrom RC, Hale PD, Kristensen EW, Kuhr WG et al (1988). Real-time characterization of dopamine overflow and uptake in the rat striatum. Neuroscience 25: 513-523.

Xu F, Gainetdinov RR, Wetsel WC, Jones SR, Bohn LM, Miller GW et al (2000). Mice lacking the norepinephrine transporter are supersensitive to psychostimulants. Nat Neurosci 3: 465-471.

Supplementary Information accompanies the paper on the Neuropsychopharmacology website (http://www.nature.com/npp) 